\title{
Study of a Rhombohedral Graphite X-Ray Filter using the sphere-in-contact model
}

\author{
Constantinos D. Zeinalipour-Yazdi, ${ }^{\mathrm{a}, \mathrm{b}, *}$ and David P. Pullman ${ }^{\mathrm{c}}$ \\ ${ }^{a}$ School of Science, University of Greenwich, Central Avenue, Chatham Maritime, Kent, ME4 4TB, UK \\ ${ }^{b}$ Department of Chemistry, University College London, 20 Gordon Street, London, WC1H 0AJ, UK \\ ${ }^{c}$ Department of Chemistry and Biochemistry, San Diego State University, 5500 Campanile Road, San \\ Diego, California 92182-1030,United States \\ *Correspondence to: c.zeinalipouryazdi@greenwich.ac.uk
}

\begin{abstract}
We have studied with the sphere-in-contact model the structure of rhombohedral graphite and found that there are molecular channels 22.7 degrees with respect to the material symmetry axis. We show via theoretical simulations with the Laue equation, Kramers equation and Duane-Hunt law that Xray radiation $(\lambda<1.34 \AA)$ that is traveling along these molecular channels would have a larger meanfree-path. This suggests that stacks of rhombohedral graphite can become effective filters for X-ray sources, such as the ones used in Laue photography and other X-ray techniques that use Bremsstrahlung.
\end{abstract}

Keywords: Rhombohedral Graphite, Sphere-in-Contact Model, X-Ray filter, Physical Molecular Models

\section{Introduction}

Graphite is a material used in many technological and commercial applications due to its chemical inertness, high melting point, strong elastic properties and unique electronic properties.[1,2] Due to its

\footnotetext{
* Corresponding author. Tel: +44 (0)208-331-9046. E-mail: c.zeinalipouryazdi@greenwich.ac.uk
} 
layered structure graphite-like carbon films have been used in various applications to enhance the tribological properties of materials.[3] In addition graphite composite materials are known to have improved mechanical properties and are broadly used in nanotechnology improved building materials.[4-6] Another commercial use of graphite is in pencil leads where different proportions of clay determine the hardness (e.g., ... 2H, H, F, HB, B, 2B, ...) as it can flake with relatively small mechanical force and decorate a paper.[7] Due to its chemical inertness, high melting point, and large heat conduction coefficient, graphite finds use as a crucible material.[8] It is also an excellent conductor of electricity along its basal plane and therefore it is used as an electrode material, which operates well even under harsh acidic conditions.[9] Graphite is broadly used as a dry lubricant and as a lubricant in metallic bearings as it can withstand high-T $\left(350{ }^{\circ} \mathrm{C}\right)$.[10] A high-tech application of graphite is in the protective material against excessive heat on the outside surface of spacecrafts [11] and as a substrate in Scanning Tunneling Microscopy (STM)[12] as it is easy to obtain contaminant free surfaces by means of cleaving the crystal along the basal plane using adhesive tape. Low mosaic-spread $\left(0.4^{\circ} \pm 0.1^{\circ}\right)$ highly-oriented-pyrolyticgraphite (HOPG) is used as a post-sample monochromator to filter out the $\mathrm{K} \beta$ radiation in $\mathrm{X}$-ray sources. [13] Graphite filter atomizers (GFA) for electrothermal atomic absorption spectrometry (ETAAS) have exhibited substantial advantages over commonly employed electrothermal vaporizers and atomizers.[14] Finally, graphite is used as the heating element in graphite furnaces [15,16] as its chemical inertness and high melting point make it ideal as the sample gasification source in atomic absorption spectrometry.[17,18] Due to these many commecial and industrial uses of graphite and the development of its method of production we attempt to find new uses of this material.

The most common method to find a new use of a material is to look at its structure. Molecular models, either simulated or physical, are therefore widely used in research, and the discovery of some of the most important molecules was based on the use of physical molecular models. Linus Pauling discovered the structure of the alpha-helix with the help of paper ribbon models that showed the alignment of hydrogen bonds [19]. Molecular models of benzene constructed by Auguste Kekulé in 1865 were also important in the discovery of the cyclic structure of carbon.[20,21] The three most common molecular models used for physical molecular and materials models are the wire-frame[22], the ball-and-stick[23], and the space-fill[24,25] models shown in Fig. 1. We have recently shown that there is one more physical molecular model, the sphere-in-contact model. [26] This new model is intermediate between the wire-frame and space-fill models and has the basic advantage that it shows the location of the electron density more accurately. 


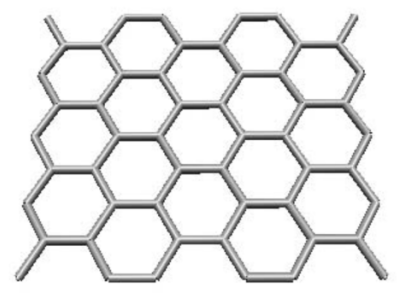

Wire-Frame

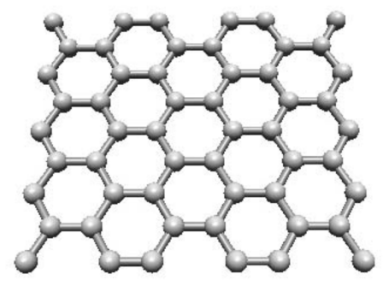

Ball-and-Stick

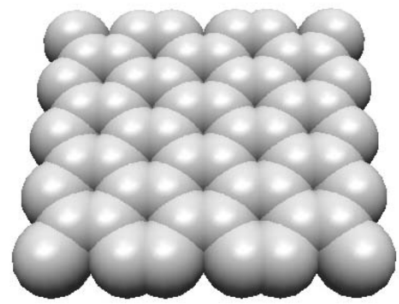

Space-Fill

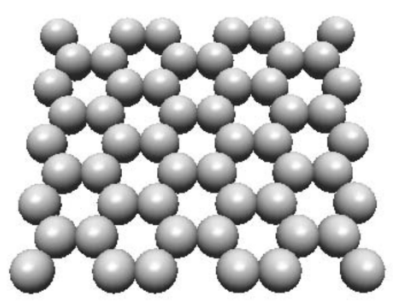

Sphere-in-Contact

Fig. 1. Various molecular models commonly used (i.e., Wire-Frame, Ball-and-Stick, Space-Fill) and the Sphere-in-Contact model for graphene nanoribbon. Structures drawn with Nanotube Modeler[27]

In this paper, we use the sphere-in-contact model design to build the structure of rhombohedral graphite and pinpoint new properties with potential applications through theoretical treatment of the spherein-contact model. Initially we derive a structure-based minimization function, $Y(r)$, which is used to calculate the radius of the carbon atoms in the sphere-in-contact model. We then describe a procedure for building physical models of graphene and graphite. We make certain observations about the interaction of electromagnetic radiation with graphite that come directly from the improved visualization of the void space in the sphere-in-contact model of rhombohedral graphite. Finally we give evidence of a potential application of rhombohedral graphite that is supported by spectral simulations of the intensity in filtered X-ray sources in transmission Laue imaging.

\section{Materials and Methods}

We enumerate a list of materials and tools needed to construct graphene and a 3-layer graphite spherein-contact models with dimensions $115 \times 235 \mathrm{~mm}$ : (1) Four hundred $14 \mathrm{~mm}$ marbles (2) Rectangular container made of paper with dimensions $>135 \times 250 \mathrm{~mm}$, (3) 3 stacks of paper notes $(100 \times 100 \times 18$ $\mathrm{mm}$ ), (4) solvent adhesive to bind the poly(methyl)-methacrylate (PMMA) sheets, (5) a10 ml syringe with hypodermic needle to dispense solvent adhesive, (6) 3 rectangular sheets of PMMA $(135 \times 250 \times 10$ $\mathrm{mm}$ ), (7) a $5 \times 2$-component (resin and hardener) epoxy resin adhesive and (8) plastic or metallic tweezers that can clamp $15 \mathrm{~mm}$ wide objects.

Dried epoxy resin glue is relatively hazard free and safe when handled with care. Uncured epoxy resin ingredients (Bisphenol-A-(epichlorohydrin)) and solvent adhesive (1-methylpropan-2-ol, dichloromethane) may cause sensitization of skin and irritation of the eyes. We recommend the use of fastdrying epoxy-glue, so that the hardening time is not more that 90 seconds. The diameters of the marbles were measured using a digital calliper, and the max-to-min width of the size distribution of glass mar- 
bles was $1 \mathrm{~mm}$. We therefore only used marbles that were in the $13.5 \pm 0.5 \mathrm{~mm}$ range, to ensure that near-perfect contact could be obtained once placed in a hexagonal close-packed array. This is critical in getting good binding between the marbles using epoxy glue that has the tendency to flow.

\section{Results and Discussion}

\subsection{Fitting the Sphere-in-contact model to crystallographic data}

In the sphere-in-contact model[28], the bond length (BL) between atoms is the sum of their atomic radii $(\mathrm{AR})$ :

$$
\mathrm{BL} \approx \mathrm{r}_{\mathrm{A}, \mathrm{AR}}+\mathrm{r}_{\mathrm{B}, \mathrm{AR}}
$$

which is in accordance of Bragg's [29] notion of the crystal structures of solids which is used in the hard-sphere model of the crystal structure of metals. We derive a minimization function, $Y(r)$ that is fitted to crystallographic data in order to determine the optimum value for the radius of the spheres, which maximises the perfect contact points between spheres. The function evaluates the pairwise nearestneighbor distance between carbon atoms given by

$$
d=\sqrt{\sum_{k=1}^{3}\left(q_{k}-p_{k}\right)^{2}}
$$

and it compares it to the distance where the carbon atoms are in perfect contact, i.e. when $\mathrm{BL}=2 r$. The ratio of the difference between $\mathrm{d}$ and $2 \mathrm{r}$, given by $(d-2 r) / 2 r$, can be positive or negative, depending on the distance between the carbons atoms and is therefore squared to yield positive values. By summing over every nearest-neighbhour distance and taking the average, we have derived a function that when minimized can yield the optimum radius of the spheres that maximizes the number of carbon pairs that are in perfect contact. The minimizing function is given by,

$$
Y(r)=\frac{1}{m} \sum_{i}^{n}\left(\frac{\sqrt{\sum_{k=1}^{3}\left(q_{k}-p_{k}\right)^{2}}}{2 r}-1\right)^{2}
$$

where $r$ is the radius of the spheres, $q_{k}$ and $p_{k}$ are the Cartesian coordinates of the centers of the atoms, $m$ is the number of pairwise nearest neighbour contacts (i.e. chemical bonds), and $n$ is the number of atoms. If this expression is rewritten for a molecular structure with Cartesian coordinates in Euclidean space of $\left(x_{1}, x_{2}, \ldots, x_{i}, x_{j}, \ldots, x_{n}\right),\left(y_{1}, y_{2}, \ldots, y_{i}, y_{j}, \ldots, y_{n}\right)$ and $\left(z_{1}, z_{2}, \ldots, z_{i}, z_{j}, \ldots, z_{n}\right)$ it is, 


$$
Y(r)=\frac{1}{m} \sum_{i, j}^{n}\left(\frac{\sqrt{\left(x_{i}-x_{j}\right)^{2}+\left(y_{i}-y_{j}\right)^{2}+\left(z_{i}-z_{j}\right)^{2}}}{2 r}-1\right)^{2}
$$

This function was implemented in a linear least-square minimization routine that found $\mathrm{r}(\mathrm{C})=0.7 \mathrm{~nm}$ for hexagonal graphite. A similar function that is parametrized for different atoms (i.e. C, H, N, O, S) can be applied via multi-variable fitting to XRD structures to obtain the sphere-in-contact radius in an arbitrary organic compound, making it possible to represent organic molecules with the sphere-incontact model. The action of this function can be seen in the following simplified derivation for the sphere-in-contact model of just two spheres. Eqn. 3 can be rewritten as the distance between two atom centers according to Fig. 2, which yields,

$$
Y(r)=\left(\frac{d}{2 r}-1\right)^{2}
$$

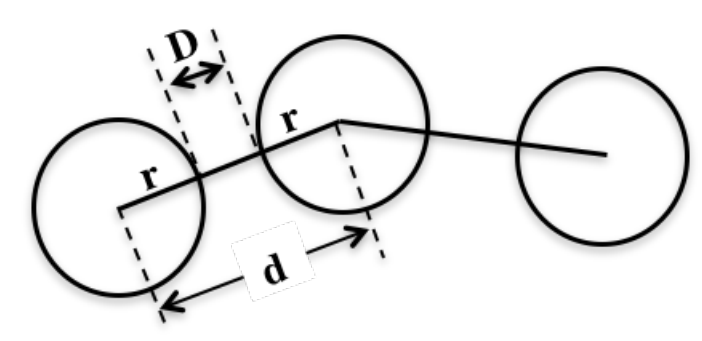

Fig 2. Scheme that shows the various variables in the sphere-in-contact model

This function has a critical value at $2 \mathrm{r}$, which based on the second derivative test, is a minimum given by

$$
\frac{\partial \mathrm{Y}(\mathrm{r})}{\partial \mathrm{r}}=-\frac{\mathrm{d}}{\mathrm{r}^{2}}\left(\frac{\mathrm{d}}{2 \mathrm{r}}-1\right)=0 \quad \therefore \quad \mathrm{d}=2 \mathrm{r} \text { and } \quad \mathrm{Y}^{\prime \prime}(2 \mathrm{r})=\frac{2}{\mathrm{r}^{2}}>0
$$

For the experimental coordinates of graphite we have used the coordinates of a single graphite layer taken from the crystallographic analysis of Bernal, who examined both natural and artificial graphite using X-ray diffractometry (XRD). The structure of graphite in his XRD refinement was found to be composed of planar layers of hexagonally-arranged rings stacked in an ABAB sequence [30]. The unit cell of hexagonal graphite along with the lattice parameters is depicted in Fig. 3a and its caption. There is another less stable form of graphite, rhombohedral graphite [31] (see Fig. 3b), which can be obtained via mechanical distortions of hexagonal graphite due to sliding of the layers [32] or for graphite grown on curved surface, such as in Kish graphite [33], produced during the manufacture of iron. [34] 


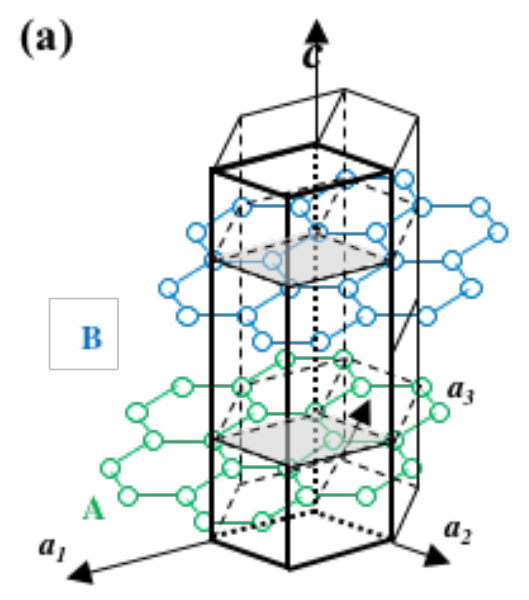

(b)

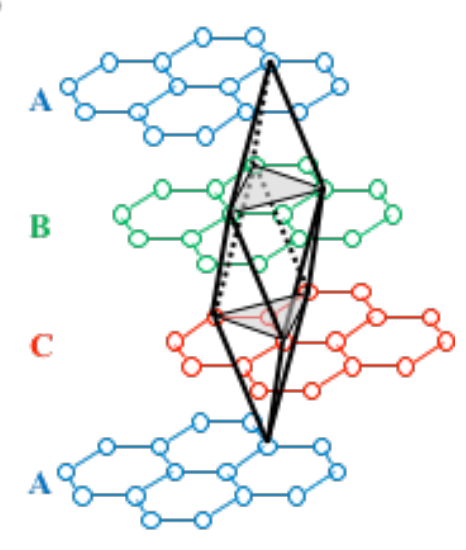

Fig. 3. Ball-and-stick models of (a) hexagonal $\left(a_{1}=a_{2}=2.45 \pm 0.03 \AA, c=6.82 \pm 0.04 \AA, \alpha=\beta=90^{\circ}\right.$ and $\left.\gamma=120^{\circ}\right)$ and (b) rhombohedral $\left(a=b=c=3.635 \AA\right.$ and $\left.\alpha=\beta=\gamma=39.49^{\circ}\right)$ structure of graphite belonging to the $\mathrm{D}_{6 h}^{4}-P 6_{3} / m m c$ and $\mathrm{D}_{3 d}^{5}-R \overline{3} m$ space groups, respectively. [35]

\subsection{Sphere-in-contact model of graphite}

In the following section we describe a procedure to construct a model of rhombohedral graphite and graphene using marbles and epoxy glue. Such a model makes possible the visualisation in order to of the molecular channels in rhombohedral graphite very easily and we describe this in the following section. In Fig. 4 the direction of the channels is shown by the red dashed line and it is clear that such channels are only present in the rhombohedral phase but absent in the hexagonal phase.

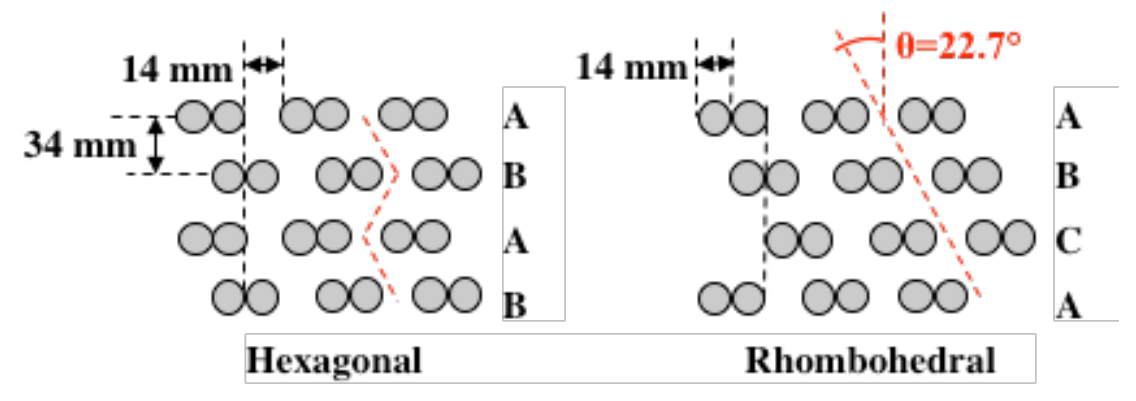

Fig. 4. Side-view schematic of stacking sequence in hexagonal (ABAB) and rhombohedral (ABCA) graphite using the sphere-in-contact model. Red dashed lines indicate hexagonal channels that extend through the structure of graphite.

When these models are built out of spheres with a diameter of about $14 \mathrm{~mm}$ then the resulting scale is $1: 10^{8}$, in which every $1 \mathrm{~cm}$ measured on the model corresponds to 1 Angstrom $(\AA)$ in the real structure. 
There is a predictive capability of the sphere-in-contact model to discover new carbon structures that are not limited to the configurational space of $\mathrm{sp}^{3}, \mathrm{sp}^{2}$ and $\mathrm{sp}$ hybridized carbon atoms as we have recently shown for the elucidation of the cap structure of single-wall carbon nanotubes (SWCNTs). [36]

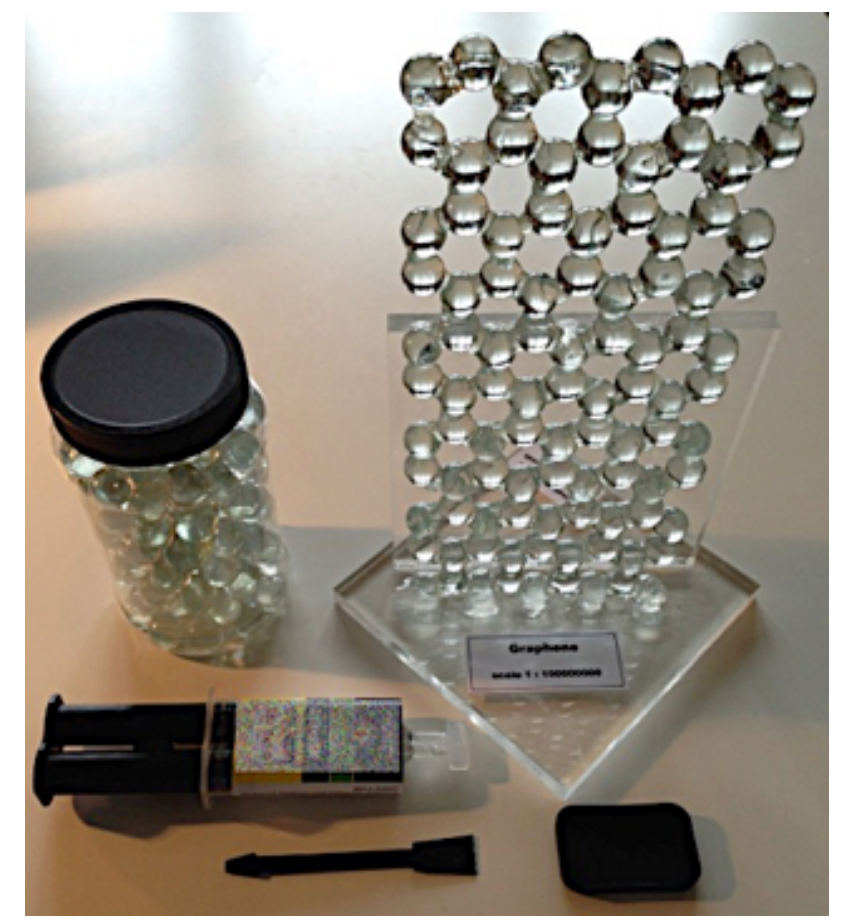

Fig. 5. Graphene model made out of marbles and epoxy glue. PMMA sheets placed in an inverse Tgeometry support the graphene structure.

The sphere-in-contact models of graphene and graphite are depicted in Fig. 5 and 6, respectively. A brief description of the procedure for building the model of rhombohedral graphite is given next and pictorially shown in Fig. 7.

Initially we placed 144 marbles of diameter $14 \mathrm{~mm}$ in a rectangular container (Fig. 6a) and moved the container sidewise until the marbles were arranged in a 2D close-packed hexagonal array (see Fig. 6b). Additional support frames are placed to hold the close-packed structure in place. 

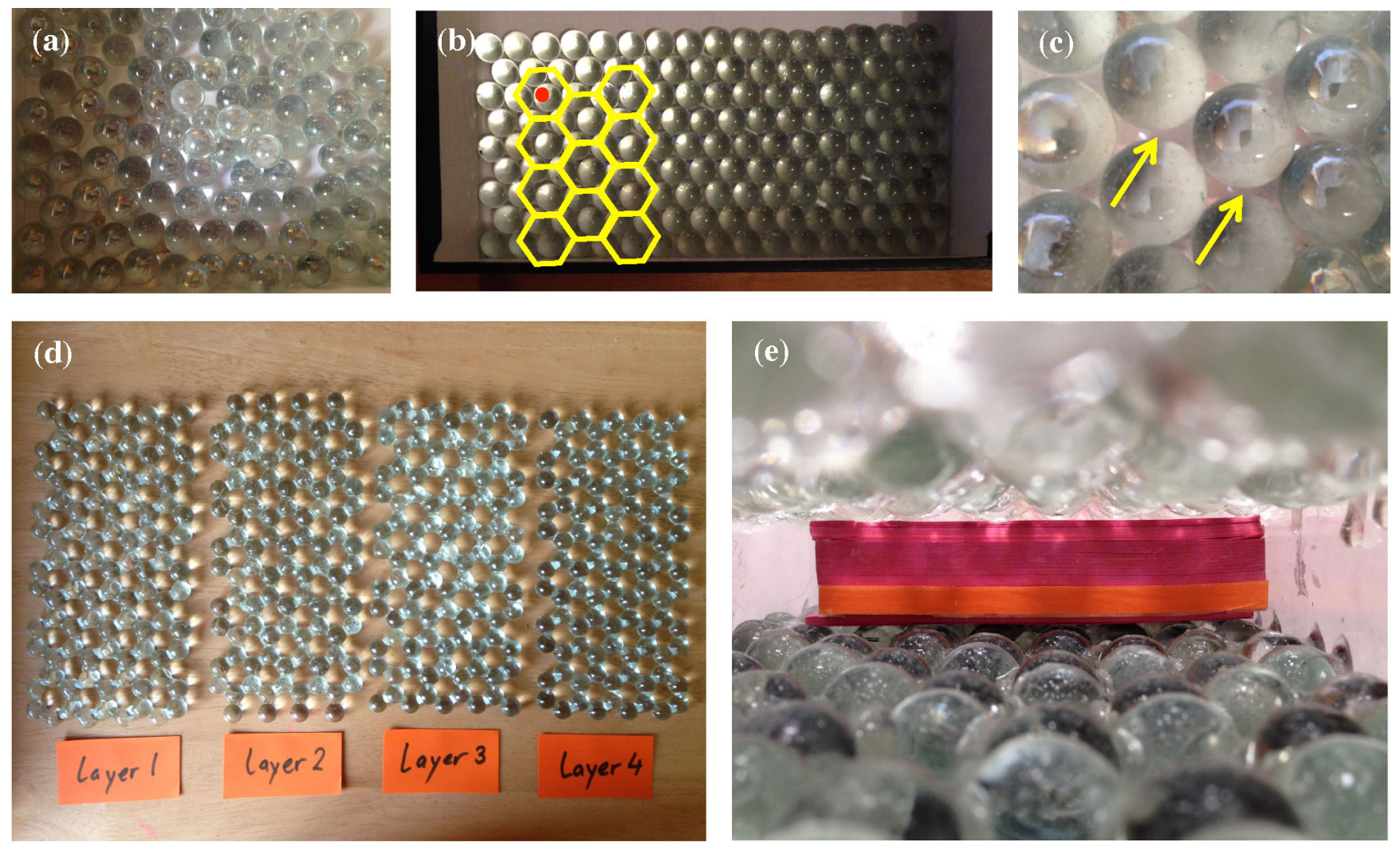

Fig. 6. Pictorial procedure for constructing rhombohedral graphite and graphene sphere-in-contact.

Depending on the size distribution of the marbles, some swapping of them in the close-packed array was necessary. This ensured good contact between the marbles and that the epoxy resin stayed at their contact point. In Fig. 6c we show that $1 \mathrm{~mm}$ caps are present in the initial hexagonal array of the marbles. We then mixed epoxy glue and hardener thoroughly by means of a disposable spatula and placed a small amount at the contact point of the marbles, so that in each hexagon, a center marble is unbound (see Fig. 6b). Once the epoxy cured (1-2 min), we used tweezers to remove the center marble of each hexagonal ring, to avoid binding of atoms that are not part of the graphene structure. After approximately $20 \mathrm{~min}$, the epoxy resin had cured, and the graphene model was ready. The graphene layer was handled with care as complete curing happens after 12 hrs. The graphene support structure can be built by placing two PMMA sheets in inverse-T geometry (see Fig. 5). With the above procedure, we built two more graphene layers shown in Figure 7. We note that the zigzag edge was different in each graphene layer so that when the layers are stacked, they would have about the same lateral dimensions with respect to the 3-layer model. 


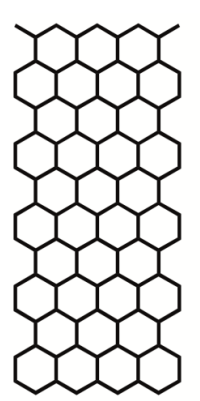

A

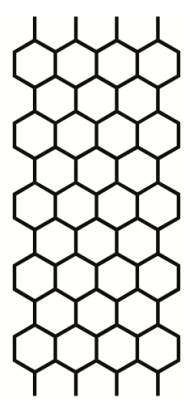

B

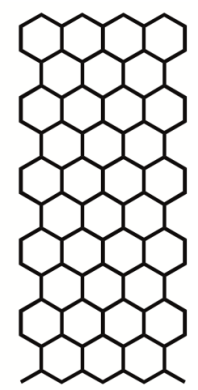

C

Fig. 7. Schematic showing each layer for a 3-layer model of graphite with ABC stacking sequence.

We then stacked graphene layers on top of one another using paper note stacks, $20 \mathrm{~mm}$ high, to adjust the distance between the layers to be $34 \mathrm{~mm}$ (see Fig. 6e). The first two graphite layers (AB) were then fixed in their position with the help of supporting PMMA sheets ( $>5 \mathrm{~mm}$ in width) as shown in Fig. 8b. Two clear PMMA sheets were brought into contact with the armchair edge of graphene layers $\mathrm{AB}$ and bound with epoxy-glue. The last step is repeated, for layer C, according to Figure 4, which shows a side-view of the stacking sequence in hexagonal and rhombohedral graphite.
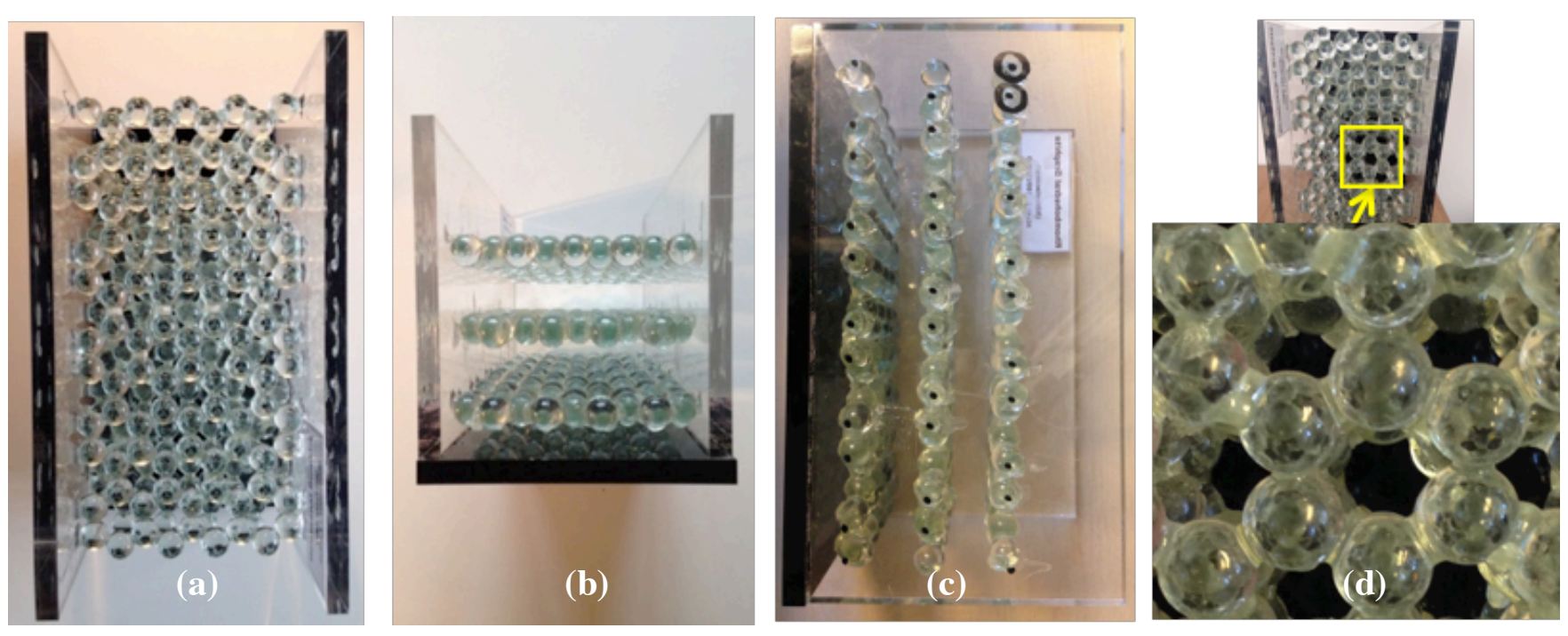

Fig. 8. (a) Top-view (b) side-view (short) (c) side-view (long) and (d) perspective view of rhombohedral graphite an inset showing the molecular channels along which electromagnetic radiation has a larger mean-free-path. Scale $1: 10^{8}$.

Once all three layers were in place, the two supporting PMMA sheets were bound to another PMMA sheet at the base (see Fig. 8b), with the use of solvent adhesive. We dispensed the solvent adhesive with a $10 \mathrm{ml}$ syringe that has a hypodermic needle, which due to capillary action forces the correct amount of adhesive between the contact surfaces of two PMMA sheets. Drying of the solvent adhesive happened in less than a minute. Note that the PMMA sheets we used had a polished edge so that the capillary action 
of the adhesive was possible. We then attached labels to the physical sphere-in-contact models to show the name and scale (e.g., rhombohedral graphite, scale $=1: 10^{8}$ ).

\subsection{Molecular channels in rhombohedral graphite}

We find that there are certain new properties of graphite observed in the sphere-in-contact model, and these are attributed to the use of proper scale and proportions. Based on these physical model it becomes obvious that diffusion through the layer is unlikely [37], except for elements with small relative atomic mass (e.g., Helium [38]), but diffusion between the layers (e.g., Lithium-cation [39]) is possible due to a gap of $2.0 \AA$ between two consecutive layers of graphite. This gap can fit small molecules, for example it fits water with its symmetry axis lying along the basal plane as the vdW radius of oxygen is $1.52 \AA$ ), which explains the exfoliation of graphene from graphite in water [40] and the diffusion of water in graphite-oxides (GO) [41]. This may also be detrimental to the lifetime of graphite-based batteries under humid conditions, which causes swelling. Furthermore, the void space in the sphere-in-contact model can explain the existence of graphite intercalation compounds (GICs) and predict which elements based on their atomic radius would fit better between the layers and how the intercalation would affect the stacking of the layers. As an example the AAA stacking sequence of $\mathrm{LiC}_{6}$ and $\mathrm{KC}_{8}$ GIC's can be predicted, as that arrangement maintains packing as dense as that of the graphite with larger interlayer cavities to accomodate $\mathrm{Li}$ and $\mathrm{K}$ atoms. There are certain channels within the lattice of rhombohedral graphite that have a cross-section of a benzene ring (along the dashed red line shown in Fig. 4) which are found at an angle of $22.7^{\circ}$, with respect to the surface normal. These channels appear in rhombohedral graphite but not in hexagonal graphite and can clearly be seen in the sphere-in-contact model of rhombohedral graphite shown by the yellow arrow in Fig. 8d. It is expected that radiation of a shorter wavelength of the channel diameter $(\mathrm{d}=1.4 \AA)$ will have a greater transmission, therefore $\lambda_{\max }<1.4 \AA$. This could find use in high-tech applications where there is the necessity to either (i) increase the penetration depth of the high frequency Bremsstrahlung band interacting with highly-oriented-pyrolitic-graphite (HOPG), (ii) enhance the transmission of X-rays with wavelengths shorter than $\lambda=1.34 \AA$ or (iii) reduce the intensity for the Bremsstrahlung continuum in X-rays reflected from HOPG. There are various places where this may have found use in commercial and high-tech applications. For example radiation that enters deeper into the bulk of graphite, rather than being reflected at its surface, would have a cooling effect to the surface of graphite and therefore reduce its possible high-T degradation. This relates to the use of graphite as the protective material in spacecrafts for heat insulation from cosmic rays and Xrays due to its very high intra-layer heat coefficient.[11] The other interesting aspect of this rhombohe- 
dral modification of the structure of graphite is that it can find use in radiation filters for the Bremsstrahlung continuum, for trasmission Laue photograph instruments. Such a filter would significantly enhance the Bremsstrahlung continuum peak with respect to higher wavelength X-rays that cause linebroadening. In the following section we calculate via Kramer simulations of the Cu X-ray source intensity the Bremsstrahlung reduction ratio of a hypothetical rhombohedral graphite filter.

\subsection{Rhombohedral graphite X-ray filter}

Typically an X-ray beam can be focused to a spot in diameter of about a micrometer and the penetration depth of a 8eV X-ray source is about $300 \mu \mathrm{m}$ for mosaic HOPG.[42] This suggestes that small crystallites of rhombohedral graphite have the appropriate dimensions to become X-ray filters and would transmit/diffract most of the X-ray beam intensity when the crystal width is between $20 \mathrm{~nm}$ to $500 \mathrm{~nm}$, which is typical for nano graphite. We have calculated using the one dimensional Laue equation for transmission photography, $n \lambda=2 \mathrm{~d}\left(\cos \left(\theta_{\text {out }}-\cos \theta_{\text {in }}\right)\right)$ [43] where $\theta_{\text {in }}$ and $\theta_{\text {out }}$ are the angle of the incident wave and the angle of the elastically scattered wave, respectively, at which angles there is constructive interference for the elastically scattered rays. For this analysis we have used the theoretical sphere-in-contact model as this can be seen in the simplified schematic depicted in Fig. 9. For a periodic surface vector between two channels of $2 \mathrm{~d}=2.836 \AA$ and $\theta_{\text {in }}=22.7^{\circ}$ we have calculated $\theta_{\text {out }}$ of the highest in intensity diffraction peak. Therefore, we assumed that there is an array of scatterers separated by a lattice vector which for the sphere-in-contact model is $2 \mathrm{~d}=2.836 \AA$ shown in Fig. 9. The scattered radiation can be examined at an angle $\theta_{\text {out }}$ if the incoming rays are in phase and the path difference is an integral multiple of $\lambda$ in order to have constructive interference.

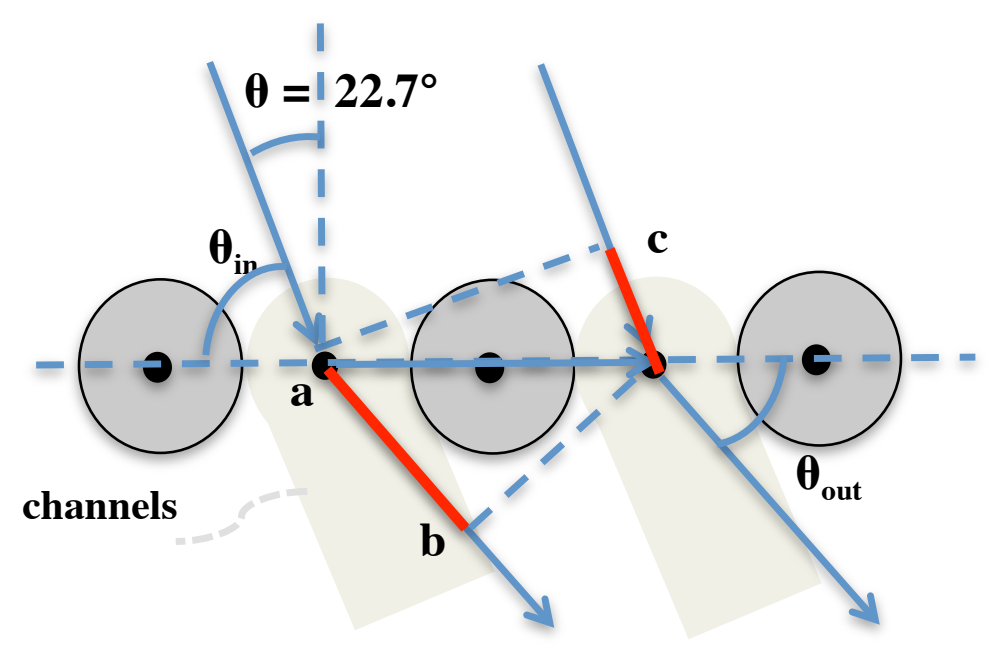


Fig. 9. Simplified schematic for derivation of Laue equation showing the incident and scatterered (in transmission mode) rays for the $1 \mathrm{D}$ case.

The path difference that the two rays have is given by (ab - cd) which based on the Laue equation [44] is given by $2 \mathrm{~d}\left(\cos \left(\theta_{\text {out }}-\cos \theta_{\text {in }}\right)=\mathrm{n} \lambda\right.$. For $\mathrm{n}=1$ where the largest intensity is expected and $\theta_{\text {in }}=$ $90-\theta=67.2^{\circ}$ we have set $\theta_{\text {out }}$ as a variable and numerically solved it for the boundary condition of $0<$ $\lambda<1.418 \AA$. This yielded that the scattered radiation beam that emerges from the ultra-thin HOPG crystal at $67.3^{\circ}<\theta_{\text {out }}<67.7^{\circ}$ and has $\lambda<1.34 \AA$. We have set this wavelength as the upper limit cut-off for the HOPG filter. The X-Ray source emission spectra have been simulated using the Kramer formula [43] given by,

$$
I(\lambda) d \lambda=K\left(\lambda / \lambda_{\min }-1\right)\left(1 / \lambda^{2}\right) d \lambda
$$

where $\mathrm{K}$ is proportional to the atomic number of the target element and $\lambda_{\min }$ is given by the DuaneHunt law [45] $\lambda_{\text {min }}=12.398 \AA / V_{K}$, where $V_{K}$ is the excitation potential of the X-ray source in $\mathrm{kV}$. The characteristic emission lines of the Copper source have been introduced by a Lorentzian function with a FWHM of $0.2 \AA$ that was centered at $\mathrm{Cu} K_{a}=1.54 \AA$ and $\mathrm{Cu} K_{\beta}=1.39 \AA$, respectively. Our simulated emission spectra from a copper X-ray source shown in Fig. 10a show that Bremsstrahlung continuum peak can have significant intensity (i.e., photon count, $I(\lambda)$ ). In Fig. $10 \mathrm{~b}$ we have calculated the $\%$ ratio of the power of X-rays that are below the cut-off wavelength $(\lambda=1.34 \AA)$ of a rhombohedral graphite filter and found that at $45 \mathrm{kV}$, almost $87.4 \%$ of the Bremsstrahlung power is in the band below the cutoff wavelength. This suggest that significant enhancement of that band could be achieved by means of such rhombohedral X-Ray filter. In particular, we find that this filter would attenuate the intense characteristic line (e.g., $\mathrm{Cu} K_{a}=1.54 \AA$ and $\mathrm{Cu} K_{\beta}=1.39 \AA$ ) and lower frequency Bremsstrahlung with $\lambda>$ $1.34 \AA$ with a reduction ratio of at least 6.6 at an excitation voltage of $45 \mathrm{kV}$. Taking into account that actual emission spectrum of copper is more concentrated at the Bremsstrahlung continuum maximum around $0.5 \AA$ this reduction ratio is a lower limit [43]. 

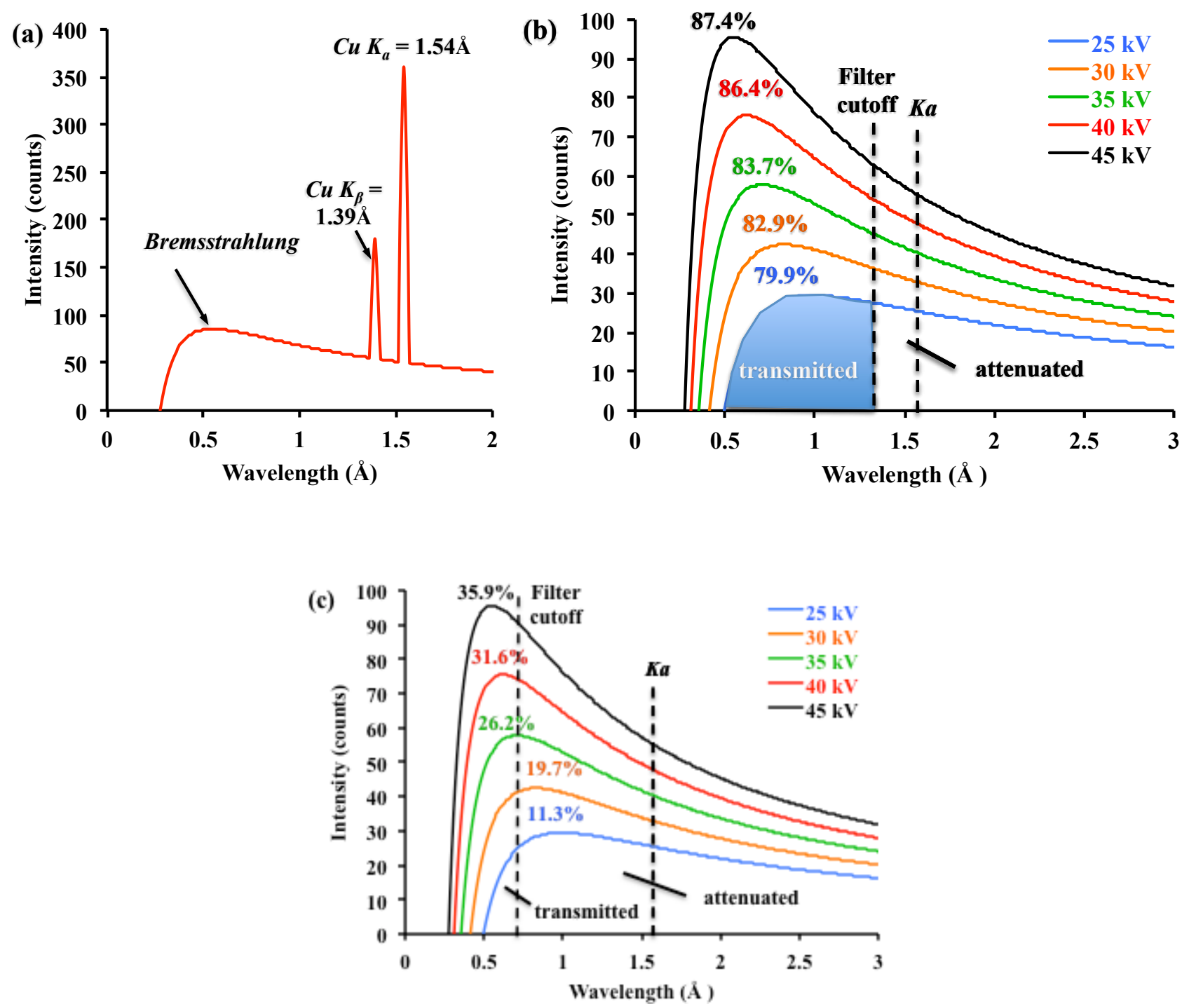

Fig. 10. (a) Simulated X-Ray emission spectrum of a Copper X-ray source at an excitation operation voltage of $40 \mathrm{kV}$. The intensity ratio of the characteristic $\mathrm{K}$ lines of copper, $\mathrm{I}(\mathrm{Ka}) / \mathrm{I}\left(\mathrm{K}_{\beta}\right)$ has been set to two for better clarity, although for copper under these conditions $\mathrm{I}(K a) / \mathrm{I}\left(K_{\beta}\right) \approx 5$. (b) Bremsstrahlung of a copper X-ray source at various excitation voltages in the range 25-45 eV indicating the percentage of transmitted versus attenuated radiation for rhombohedral graphite. (c) Bremsstrahlung of a copper Xray source at various excitation voltages in the range $25-45 \mathrm{eV}$ indicating the percentage of transmitted versus attenuated radiation for hexagonal graphite.

According to Fig. $10 \mathrm{~b}$ which shows the X-Ray beam intensity that is transmitted (e.g. $79.9 \%$ at $25 \mathrm{kV}$ ) at various excitation voltages based on the simulations, the intensity of the attenuated X-Ray beam can be preserved by as much as $87.4 \%$ in the rhombohedral graphite filter although the intense $C u K_{\alpha}$ and $\mathrm{Cu} K_{\beta}$ has been effectively filtered out. In Fig. $10 \mathrm{c}$ we compare the analogous hexagonal graphite filter 
which shows significantly lower transmission/attenuation percentages. This suggests that a rhombohedral graphite filter should in principle work. This should enhance the resolution of Laue photographs in principle. It is therefore evident that on a theoretical basis a rhombohedral graphite-based X-Ray filter is possible and should become the topic of further investigation.

\section{Conclusion}

In this study we have constructed a sphere-in-contact model of the crystal structure of rhombohedral graphite graphene in which every atom is represented by a sphere of covalent radius. In the graphite models build we observe channels that have a cross-section of a benzene ring oriented at $\theta=$ $22.7^{\circ}$ with respect to surface normal, that appear in rhombohedral graphite but not in hexagonal graphite. The physical properties of graphite are discussed in detail with reference to this model and a detailed procedure for its construction is given. We then show via theoretical simulations with the Laue equation, Kramers equation and Duane-Hunt law that X-ray radiation $(\lambda<1.34 \AA)$ that is travelling along these channels would a larger mean-free-path and that stacks of rhombohedral graphite can become effective filters for X-ray sources, such as the ones used in Laue photography.

\section{Declaration of Competing Interest}

The authors declare that there is no conflict of interest.

\section{Acknowledgement}

The authors would like to acknowledge Dr. David Scanlon from University College London for offering comments on the manuscript.

\section{References}

[1] M. Murugan, R.M. Kumar, A. Alsalme, A. Alghamdi, R. Jayavel, Chem. Phys. Lett. 650 (2016) 35 .

[2] H.J. Kim, C.-S. Yang, H. Jeong, Chem. Phys. Lett. 644 (2016) 288.

[3] D. Dong, B. Jiang, H. Li, Y. Du, C. Yang, Appl. Surf. Sci. 439 (2018) 900.

[4] H. Wang, Z. Tao, X. Li, X. Yan, Z. Liu, Q. Guo, Appl. Surf. Sci. 439 (2018) 488.

[5] H. Wang, S. Wang, W. Lu, M. Li, Y. Gu, Y. Zhang, Z. Zhang, Appl. Surf. Sci. 442 (2018) 170.

[6] R. Hu, T. Furukawa, X. Wang, M. Nagatsu, Appl. Suf. Sci. 416 (2017) 731.

[7] C.D. Zeinalipour-Yazdi, D.P. Pullman, J. Phys. Chem. B 110 (2006) 24260.

[8].

[9] T. Sagara, K. Niki, Langmuir 9 (1993) 831.

[10] .

[11] D.G. Zimcik, C.R. Maag, J. Spacecraft Rockets 25 (1988) 162.

[12] C.D. Zeinalipour-Yazdi, D.P. Pullman, Chem. Phys. 348 (2008) 233.

[13] R. Arnhold, S. Kauter, Cryst. Res. Technol. 23 (1988) 1187. 
[14] D.A. Katskov, Spectrochim. Acta Part B At. Spectrosc. 62 (2007) 897.

[15] J. Pedro, J. Stripekis, A. Bonivardi, M. Tudino, Spectrochim. Acta Part B At. Spectrosc. 107 (2015) 152.

[16] A. Brandt, K. Leopold, Spectrochim. Acta Part B At. Spectrosc. 150 (2018) 26.

[17] F. Cacho, L. Machynak, M. Nemecek, E. Beinrohr, Spectrochim. Acta Part B At. Spectrosc. 144 (2018) 63.

[18] J.O. Vinhal, R.J. Cassella, Spectrochim. Acta Part B At. Spectrosc. 151 (2019) 33.

[19] L. Pauling, R.B. Corey, H.R. Branson, P. Natl. Acad. Sci. USA 37 (1951) 205.

[20] A. Kekulé, B. Soc. Chim. Fr. 3 (1865) 98.

[21] A. Kekulé, Ann. der Chem. and Pharm. 137 (1866) 129.

[22] A.S. Dreiding, Helv. Chim. Acta 42 (1959) 1339.

[23] W.D. Ollis, Proceedings of the Royal Institution of Great Britain 45 (1972) 1.

[24] R.B. Corey, L. Pauling, Rev. Sci. Instr. 24 (1953) 621.

[25] W.L. Koltun, Space Filling Atomic Units and Connectors for Molecular Models, US, 1965.

[26] C.D. Zeinalipour-Yazdi, D.P. Pullman, C.R.A. Catlow, J. Molec. Model. 22 (2016) 40.

[27] S. Melchor, J.A. Dobado, J. Chem. Inf. Comput. Sci. 44 (2004) 1639.

[28] C.D. Zeinalipour-Yazdi, D.P. Pullman, C.R.A. Catlow, Journal of Molecular Modeling 22 (2016) 40.

[29] W.L. Bragg, Philos. Mag. 40 (1920) 169.

[30] J.D. Bernal, Proc. Phys. Soc. A 106 (1924) 749.

[31] H. Lipson, A.R. Stokes, Proc. Roy. Soc. A 181 (1942) 101.

[32] E.J. Freise, A. Kelly, Philos. Mag. 8 (1963) 1519.

[33] A.N. Roviglione, J.D. Hermida, Procedia Mat. Sci. 8 (2015) 924.

[34] B.T. Kelly, Physics of Graphite, Applied Science Publishers, London, 1981.

[35] C.D. Zeinalipour-Yazdi, PhD Thesis, University of California, San Diego, CA, Digital

Dissertations, 2006.

[36] C.D. Zeinalipour-Yazdi, E.Z. Loizidou, Carbon 115 (2017) 819.

[37] A. Roy, M.K.P. Kumar, C. Srivastava, Chem. Phys. Lett. 646 (2016) 158.

[38] H. Atsumi, J. Nucl. Mat. 179-181 (1991) 227.

[39] K. Persson, V.A. Sethuraman, L.J. Hardwick, Y. Hinuma, Y.S. Meng, A. van der Ven, V.

Srinivasan, R. Kostecki, G. Cede, J. Phys. Chem. Lett. 1 (2010) 1176.

[40] K. Parvez, Z.-S. Wu, R. Li, X. Liu, R. Graf, X. Feng, K. Müllen, J. Am. Chem. Soc. 136 (2014) 6083.

[41] V.A. Smirnov, V.P. Vasil'ev, N.N. Denisov, Y.V. Baskakova, V.A. Dubovitskii, Chem. Phys. Lett. 648 (2016) 87.

[42] U. Zastrau, C.R.D. Brown, T. Döppner, S.H. Glenzer, G. Gregori, H.J. Lee, H. Marschner, S. Toleikis, O. Wehrhana, E. Förster, JINST 7 (2012) 9015.

[43] D. Laguitton, W. Parrish, X-Ray Spectrom. 6 (1977) 201.

[44] W. Friedrich, P. Knipping, M. Laue, Annalen der Physik 346 (1913) 971.

[45] W. Duane, F.L. Hunt, Phys. Rev. 6 (1915) 166. 